\title{
Irreducible Elbow Dislocation Associated with Hill-Sachs-like Lesion over the Capitellum
}

\author{
Hung-Kai Weng ${ }^{1,2,3}$, Wei-Lun Chang ${ }^{1,3}$, Ming-Long Yeh ${ }^{4}$, Wei-Ren $\mathrm{Su}^{1}$, Kai-Lan Hsu ${ }^{1,4,5 \rrbracket}$ \\ ${ }^{1}$ Department of Orthopedic Surgery, National Cheng Kung University Hospital, College of Medicine, National Cheng Kung University, ${ }^{2}$ Institute of Basic Medical \\ Sciences, College of Medicine, National Cheng Kung University, Tainan, ${ }^{3}$ Division of Orthopedics, Department of Surgery, National Cheng Kung University Hospital \\ Dou Liou Branch, College of Medicine, National Cheng Kung University, Yunlin, ${ }^{4}$ Department of Biomedical Engineering, National Cheng Kung University, ${ }^{5}$ Division \\ of Traumatology, National Cheng Kung University Hospital, College of Medicine, National Cheng Kung University, Tainan, Taiwan
}

Irreducible dislocation of the elbow is an uncommon event. We present the case of a posterolateral elbow dislocation after a fall injury in a 67-year-old woman. A closed reduction performed in the emergency department was unsuccessful since the limited passive range of motion resulted in difficulty to perform longitudinal traction and flexion. Computed tomography images showed that the posterolateral aspect of the capitellum was impacted by the tip of the coronoid process, thus appearing similar to the Hill-Sachs lesion in the humeral head. Subsequent open reduction of the elbow revealed the dislocation to be irreducible since the tip of the coronoid process had wedged into a triangular Hill-Sachs-like lesion in the capitellum. The joint was reduced by providing distal traction on the forearm, and main fragments were disengaged using digital pressure. At the 3-month follow-up, the patient reported no dislocations, and had an acceptable range of motion. Thus, we propose that to avoid iatrogenic injury to the joint or other nearby structures, irreducible dislocations should not be subjected to repeated manipulation.

(Clin Shoulder Elbow 2019;22(1):37-39)

Key Words: Hill-Sachs lesions; Fracture dislocation; Avulsion fracture

Elbow dislocations constitute $10 \%$ to $25 \%$ of all injuries to the elbow, and are usually caused by a fall on an outstretched hand. ${ }^{1)}$ Most elbow dislocations are treated with closed reduction. Few literature reports are available on irreducible elbow dislocations, and these mostly describe soft-tissue interposition. $^{2,3)}$ In the current case report, we present the rare case of a 67-year-old woman with an irreducible posteromedial elbow dislocation associated with a Hill-Sachs-like lesion over the capitellum.

\section{Case Report}

A 67-year-old woman presented to the emergency department with acute painful swelling over the left elbow resulting from a fall on an outstretched hand. On inspection, we found a posterolateral olecranon protrusion. Radiographs revealed a posterolateral dislocation of the proximal radius and ulna relative to the distal humerus, and an avulsion fracture of the lateral humeral epicondyle (Fig. 1A, B). Closed reduction was subsequently attempted under sedation in the emergency department. However, the elbow joint appeared to be locked and had a limited range of motion $\left(60^{\circ}-90^{\circ}\right)$, thereby making application of longitudinal traction and flexion difficult. The elbow joint could, therefore, not be reduced, since excessive manual manipulation would result in additional damage to other structures.

The patient underwent computed tomography (CT), which revealed a posterolateral dislocation with a bony fragment avulsed from the lateral humeral epicondyle. In addition, we

Received August 29, 2018. Revised October 26, 2018. Accepted October 30, 2018.

Correspondence to: Kai-Lan Hsu

Department of Orthopedic Surgery, National Cheng Kung University Hospital, College of Medicine, National Cheng Kung University, No.138, Sheng Li Road, Tainan 704, Taiwan

Tel: +886-6-2353535, Fax: +886-6-2766189, E-mail: dulendulen@gmail.com, ORCID: https://orcid.org/0000-0002-7576-2486

IRB approval: National Cheng Kung University Hospital (No. A-EC-107-007).

Financial support: None. Conflict of interests: None. 

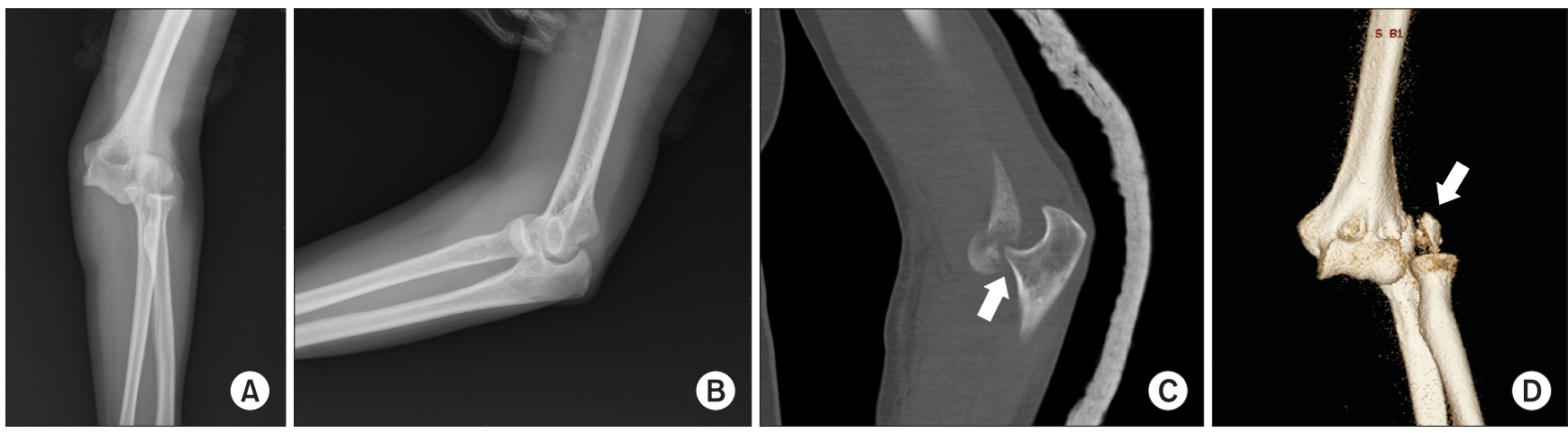

Fig. 1. (A) Anteroposterior radiograph of the left elbow: an avulsion fracture of the lateral humeral epicondyle is noted. (B) Lateral radiograph of the left elbow. (C) Computed tomography (CT) shows posterolateral aspect of the capitellum is impacted by the tip of the coronoid process (arrow). (D) Three-dimensional CT shows a posterolateral dislocation with a bony fragment avulsed from the lateral humeral epicondyle (arrow).

found that the posterolateral aspect of the capitellum was impacted by the tip of the coronoid process (Fig. 1C, D). Open reduction of the elbow was subsequently performed, adopting the Kocher approach in the supine position. The joint space was directly exposed via the avulsion fracture gap. The dislocation was irreducible since the tip of the coronoid process had wedged into a triangular defect in the capitellum (Fig. 1C); no buttonhole entrapment was noted. The joint was reduced through distal traction of the forearm, and the major fragments were disengaged using digital pressure. Following joint reduction, No. 2 Ethibond (17 mm, 1/2 circle tapercut, Ethibond 2-0; Ethicon, Somerville, NJ, USA) was applied for attaching the avulsed fragment to the lateral supracondyle. Fluoroscopy confirmed concentric reduction of the joint, and the elbow remained stable through a full range of motion (Fig. 2).

Postoperatively, the patient was placed in a long arm splint with the elbow joint at a $90^{\circ}$ flexion. Active and passive rangeof-motion exercises were commenced at 1 week after surgery. There was no incidence of redislocation, instability, or neurological symptoms. The splint was removed at 6 weeks. At the 3-month follow-up, the patient was pain-free, and her motion ranged from $15^{\circ}$ to $130^{\circ}$ of flexion.

\section{Discussion}

Dislocation of the elbow joint is less common than dislocation of the shoulder, and $90 \%$ of dislocations occur with posterior or posterolateral displacement of the forearm relative to the distal humerus. ${ }^{1)}$ Following appropriate analgesia, most elbow dislocations can be treated by closed reductions, by applying traction to the forearm and countertraction to the upper arm. In rare cases, elbow dislocations are irreducible and require open reduction.

Most irreducible elbow dislocations are caused by interposition of soft tissues, such as brachial muscles and collateral ligaments. These dislocations are also caused by the radial head protruding or buttonholing through the lateral collateral ligament
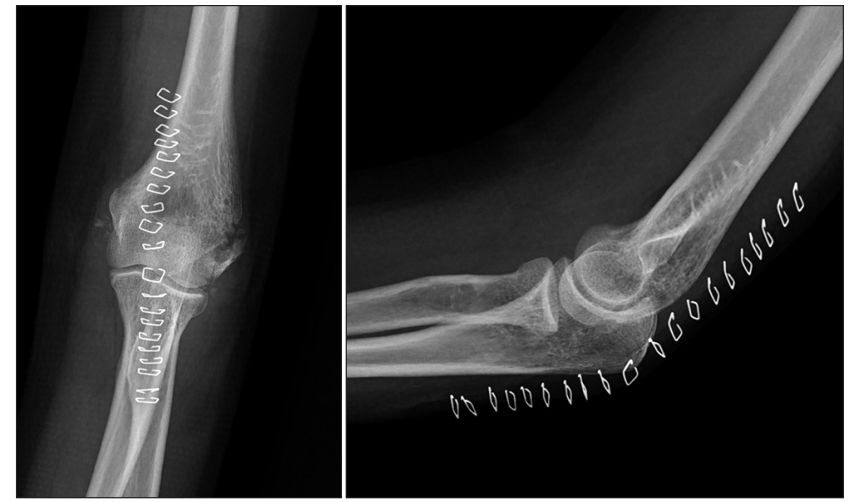

Fig. 2. Anteroposterior radiograph and lateral radiograph of the left elbow after open reduction.

complex.. An avulsion fracture is another cause of irreducible elbow dislocation, and the avulsed fragment may be entrapped in the joint space, thus making reduction unavailable." However, in the present case, we observed no soft-tissue interposition intraoperatively, or any fracture fragments entrapped in the joint space. In fact, the reduction was blocked by the bony impaction on the posterolateral aspect of the capitellum.

Bony impactions are insults to the subchondral bone during a joint dislocation, and are usually mild and difficult to detect on imaging. However, if dislocations are neglected or repeated, the lesion may enlarge and block the direction of reduction. Irreducible dislocations related to bony impact lesions are reported in shoulder ${ }^{5}$ and lateral patella ${ }^{6}$ dislocations. Osborne and Cotterill ${ }^{7)}$ reported the presence of a bony impact lesion in the capitellum following elbow dislocation. This lesion resulted from the shear force exerted by the radial head onto the posterior capitellum during elbow hyperextension. The lateral ulnar collateral ligament gets damaged after the repeated engagement of the radial head in bony defects, resulting in chronic instability. ${ }^{8)}$ Some differences were observed between the bony lesion in our case and that reported by Osborne and Cotterill.7) First, 
the osteoporotic capitellum was impacted by the sharp coronoid process instead of the blunt radial head; the deep bony defect then blocked the direction of reduction. Second, the shear force resulted in an avulsion fracture of the lateral epicondyle instead of a lateral ulnar collateral ligament injury.

A lateral humeral epicondyle avulsion fracture associated with elbow dislocation is also rare, and has been described in only few case reports." This fragment is usually avulsed by the extensor tendons, muscles, and lateral collateral ligament complex. Non-displaced fractures of the lateral humeral epicondyle usually heal after a short immobilization period. Surgical intervention is indicated if the fragment is incarcerated within the elbow joint or if it causes a block to extension or instability. In the present case, we simultaneously performed open reduction of the elbow joint and fixation of the displaced avulsion fracture to avoid any joint instability. Compared with the lateral ulnar collateral ligament injury reported by Osborne and Cotterill, ${ }^{7}$ the repair and healing processes of avulsion fractures are easier; no further instability was noted in the presented case.

Both magnetic resonance imaging (MRI) and CT are implemented to find the cause of irreducible dislocation. MRI identifies the soft tissues involved and facilitates accurate preoperative planning. ${ }^{2)}$ However, $\mathrm{CT}$ is more accessible, less expensive, and is useful in demonstrating intraarticular extension of fractures or the distribution of small fracture fragments. Because of the presentation of an avulsion fracture and the locked response while performing reduction in this case, we applied CT rather than MRI to survey the possible lesion blocking the reduction. CT findings are important since they help prevent further closed reduction attempts, which may otherwise damage the joint by increasing the amounts of bony impactions.

In conclusion, irreducible posterolateral elbow dislocations with an impacted bony lesion are rare. To prevent iatrogenic injury to the joint or other nearby structures, repeated manipulations of dislocations unavailable to be reduced should not be attempted. Failure to reduce dislocations should prompt further imaging such as CT, to define the injury and identify the cause of obstruction to reduction, before further manipulation or operative intervention.

\section{Acknowledgements}

This manuscript was edited by Wallace Academic Editing.

\section{References}

1. Cohen MS, Hastings $\mathrm{H}$ 2nd. Acute elbow dislocation: evaluation and management. J Am Acad Orthop Surg. 1998;6(1):1523.

2. Kim SJ, Ji JH. Irreducible posteromedial elbow dislocation: a case report. J Shoulder Elbow Surg. 2007;16(6):e1-5. doi: 10.1016/j.jse.2006.09.013.

3. Fenelon C, Zafar MM, Sheridan GA, Kearns S. Irreducible posterolateral elbow dislocation: a rare injury. BMJ Case Rep. 2016;2016:bcr2016217967. doi: 10.1136/bcr-2016-217967.

4. Pouliart N, De Boeck H. Posteromedial dislocation of the elbow with associated intraarticular entrapment of the lateral epicondyle. J Orthop Trauma. 2002;16(1):53-6.

5. Davies MB, Rajasekhar C, Bhamra MS. Irreducible anterior shoulder dislocation: the greater tuberosity Hill-Sachs lesion. Injury. 2000;31(6):470-1. doi: 10.1016/S0020-1383(00)000164.

6. Feibel RJ, Dehghan N, Cwinn AA. Irreducible lateral patellar dislocation: the importance of impaction fracture recognition. J Emerg Med. 2007;33(1):11-5. doi: 10.1016/j.jemermed. 2006.12.033.

7. Osborne G, Cotterill P. Recurrent dislocation of the elbow. J Bone Joint Surg Br. 1966;48(2):340-6.

8. Jeon IH, Min WK, Micic ID, Cho HS, Kim PT. Surgical treatment and clinical implication for posterolateral rotatory instability of the elbow: Osborne-Cotterill lesion of the elbow. J Trauma. 2011;71(3):E45-9. doi: 10.1097/TA.0b013e3182095c8a.

9. Ando A, Hagiwara Y, Koide M, Yamashiro M, Matsuda M, Itoi E. Lateral dislocation of the elbow with concomitant lateral epicondyle fracture: a case report and review of the literature. J Orthop Sci. In press, available on 2 Feb 2017. doi: 10.1016/ j.jos.2016.12.028. 